\title{
Una tensión productiva: América Latina en las páginas de Historia y Sociedad
}

\author{
A Productive Tension: Latin America \\ through the Pages of Historia y Sociedad
}

\author{
Jaime Ortega Reyna \\ (D) 0000-0002-8582-1216 \\ Universidad Autónoma Metropolitana \\ Unidad Xochimilco, México \\ jaime_ortega83@hotmail.com
}

Resumen: A partir de 1965 el pensamiento marxista auspiciado por el Partido Comunista mexicano comenzó un proceso de renovación que se encontró con una tradición de pensamiento proveniente de la influencia soviética en las ciencias sociales. La revista Historia y Sociedad en su primera época expresó de mejor forma la tensión que generó el proceso de renovación al interior del comunismo mexicano y la persistencia de coordenadas soviéticas en las ciencias sociales. En el texto que presentamos a continuación se explora el conjunto de tensiones que fueron desarrollándose a partir del estudio de distintos temas referidos a América Latina.

Palabras clave: historia; marxismo; América Latina; intelectuales; revistas.

Abstract: Beginning in 1965, Marxist thought under the auspices of the Mexican Communist Party began a process of renewal that encountered a tradition of thought stemming from Soviet influence in the social sciences. During its initial period, Historia y Sociedad more effectively expressed the tension generated by the process of renewal within Mexican communism and the persistence of Soviet coordinates in the social sciences. The text below explores the set of tensions developed on the basis of the study of various topics related to Latin America. 
Key words: history; marxism; Latin America; intellectuals; journals.

Fecha de recepción: 23 de enero de 2017 Fecha de aprobación: 13 de enero de 2018

$\mathrm{E}$ n tiempos recientes se ha profundizado en una línea de investigación al interior de los estudios latinoamericanos cuyo principal eje de articulación es un género particular de las publicaciones periódicas: las revistas. Regina Crespo (2010), una de las principales estudiosas de esta temática, ha señalado el potencial de su abordaje como objeto de estudio indicando que: "entre las nuevas líneas de investigación que se abren en el estudio de las revistas, sobresale una que posee un especial interés para la historia intelectual y política latinoamericana: la formación y el mantenimiento de redes intelectuales transfronterizas" (p. 9). Efectivamente, muchas publicaciones periódicas cumplieron con la función señalada por la estudiosa brasileña, al ser un vehículo intelectual y político que permitió desbordar fronteras y concepciones tradicionales de lo nacional, lo regional y lo mundial. Si bien esta valoración general es válida, es pertinente colocar algunos puntos de especificidad para la comprensión de lo que aquí se abordará. Así, para el caso regional Alexandra Pitta (2013) ha señalado que el estudio de este tipo de publicaciones: "parte de la premisa de que estas publicaciones tienen un papel fundante de la cultura y la política en América Latina y por lo tanto, se busca observar el lugar que ocupa América Latina 'como tema filosófico, histórico y coyuntural', en estas revistas" (p. 57). Esto nos interpela de manera inmediata, pues una noción de América Latina se juega en el planteamiento de la revista que analizaremos.

Sin embargo, no es sólo una pretensión exclusiva de la dimensión cultural lo que animará la aparición de publicaciones periódicas. El ánimo de intervenir políticamente una realidad específica es un sello distintivo de las revistas deudoras de la tradición intelectual heredada por Karl Marx. Para el caso específico de las corrientes marxistas ha sido Horacio Tarcus (2016) quien ha señalado la importancia de su estudio: "El foco que los grandes relatos ponían en los autores individuales se refracta ahora en un haz que busca iluminar la producción de los colectivos intelectuales; por ejemplo, las revistas: las culturales en general y las marxistas en particular han constituido medios privilegiados en la creación de los marxismos latinoamericanos" (p. 69). En el 
caso que desarrollaremos a continuación abordaremos la forma en que el conjunto abigarrado que denominamos América Latina fue presentado en una revista de corte marxista producida en México a mediados de los años sesenta.

Así, la aparición de la revista Historia y Sociedad supuso un proceso de renovación radical en el discurso marxista en México. Su existencia se debe a la confluencia tanto del impulso de las transformaciones que acontecían en la URSS (tras el XX Congreso del partido soviético en 1956 y el comienzo del "deshielo") como de las que sucedían al seno del Partido Comunista Mexicano (РСM) que pretendía dejar atrás los peores rastros del stalinismo de la mano de una nueva dirigencia encabezada por Arnoldo Martínez Verdugo. Aquella renovación política del partido se vio reflejada años después en el proceso de disolución del PCM (a principios de los años ochenta) y su fusión con otras fuerzas políticas y sociales que adoptaron la bandera de la democracia como medida fundamental para obtener objetivos de tipo socialista, lo que también dio pie a una transformación de la cultura política de la izquierda mexicana. A este proceso Massimo Modonesi (2003) lo ha denominado "la crisis histórica de la izquierda socialista”, pues expresa la pérdida de centralidad del horizonte político tradicional y su radical transformación.

De manera que fue el periodo dentro del cual surgió la revista el que permitió la superación de las décadas más difíciles del PCM, que había sido víctima tanto de la represión estatal como de su propia inercia stalinista, llena de purgas, expulsiones, sectarismos y múltiples conflictos. En suma, los años de inicio y desarrollo de Historia y Sociedad marcan claros indicios de una apertura del marxismo hacia otras formas del pensamiento, incluidas algunas que habían sido prohibidas o relegadas al seno de la propia tradición.

Por tanto, en este trabajo queremos explorar el vector latinoamericanista que se jugó en Historia y Sociedad durante su primera época (1965-1970), partiendo de la hipótesis de que se trata de un proceso contradictorio que podríamos denominar de tensión productiva, en tanto que se expresaron signos de renovación y de apertura, a la vez que también existió una fuerte reproducción de certezas ancladas en la tradición comunista, en su expresión regional y en la compartida con la matriz soviética. Con ello queremos marcar un distanciamiento con la reducción interpretativa que ha hecho de la revista un órgano absolutamente dependiente de la embajada soviética a lo largo de su primera etapa de existencia. Esto es crucial, toda vez que permite establecer los distintos puntos desde los que la revista intentó innovar su discurso. Aunque indudablemente el momento no favoreció un margen de acción amplio ni 
siquiera a nivel local en donde la represión era una constante, mucho menos a nivel internacional en donde la guerra fría determinó los vaivenes de la corriente comunista a nivel internacional.

En primer lugar, explicaremos la especificidad de la aparición y desarrollo de Historia y Sociedad, considerando su sitio en el contexto del marxismo producido en México y su contribución en las transformaciones en el seno del PCM. En segundo lugar, nos remitiremos a los temas que nos parecen anclados en la tradición comunista aún dependiente de la Unión Soviética, es decir, tópicos que aparecen recurrentemente en los textos y que podemos señalar como ejemplo de una continuidad de la línea de trabajo de aquella tradición. Y, en tercer lugar, expondremos materiales que consideramos intentos de renovación interpretativa, por tema y enfoque, como muestra de las confluencias de su tiempo. Este conjunto, permitirá sostener la existencia de un debate que pese a quedar irresoluto en su época, generó un efecto productivo que se tensionó entre la legitimidad del Estado soviético con su ideología y los intentos de renovación de la misma. A pesar de que dicho efecto es ausente en las expresiones públicas de los comunistas mexicanos, al menos en la región.

En efecto, conviene subrayar que continuidad y renovación no son de pertenencia nacional, por lo que, se han enunciado temas novedosos para la tradición marxista mexicana por parte de autores de origen soviético o ruso. De igual manera, es necesario indicar que la continuidad del pensamiento de aquel poder imperial no ha tenido impedimentos para reproducirse en la escritura de un autor de alguna de las nacionalidades latinoamericanas. La elección de los autores no se da de acuerdo con su nacionalidad, sino a la proyección temática, centrada en América Latina.

Por esto, nos concentraremos sólo en la primera época de Historia y Sociedad, enfocándonos así en temas de interés histórico (sólo en algún momento referiremos a un par de textos que podrían ser considerados de exclusividad teórica) y descartaremos los documentos en donde se explora la situación de México, justamente para recalcar el profundo y muy arraigado carácter latinoamericanista que tuvo la revista desde su nacimiento. En atención a nuestro objetivo, también dejaremos de lado las consideraciones sobre el "modo de producción asiático", del que, sin embargo, nos interesa mencionar que configuró en gran medida el proyecto de la revista, lo que además de confirmar que el carácter latinoamericanista de la revista convivió con estos otros elementos, permite identificar uno de los puntos más tirantes de la publicación. 


\section{EL NACIMIENTO DE HISTORIA Y SOCIEDAD}

Sólo en los años sesenta es posible pensar el nacimiento de una revista como Historia y Sociedad, esto debido a que el proceso de renovación al interior del comunismo mexicano que se labró de manera lenta, mostró hasta 1982 las máximas consecuencias de aquel ánimo de apertura. En este contexto, la figura de Arnoldo Martínez Verdugo, un intelectual y dirigente comunista crítico, de amplias miras hacia la cuestión democrática y con preocupaciones que lo llevaron a buscar la independencia del partido soviético, ha sido destacada por numerosos personajes con respecto a los aires de renovación.

Así, una estudiosa del fenómeno comunista en México afirma: "Como hemos señalado, tras 20 años de crisis, sumido en el mayor dogmatismo y marginalidad, el PCM recuperó con el nuevo grupo dirigente surgido a principios de los años sesenta una postura propia que poco a poco se fue definiendo, no sin resistencias internas, con un sentido independiente frente a la política soviética y la situación del mundo socialista de entonces" (Concheiro, 2007, p. 555). Efectivamente, al pasar los años el PCM no sólo labró una visión distinta de la política local (centrando el problema de la democracia como el más importante para desentrañar el dilema del sistema político nacido al calor de la revolución mexicana), sino que también tejió de manera distinta su relación con la Unión Soviética.

La época en que nació Historia y Sociedad ha sido tipificada en un estudio de gran valía del historiador Barry Carr (1996) como "el nacimiento de una nueva izquierda", en la que confluyeron movilizaciones campesinas (Fuente, 2016), el Movimiento de Liberación Nacional, los impulsos de la revolución cubana y nuevos aires de rebeldía entre la juventud. Todos estos elementos ejercieron una fuerte presión sobre las concepciones imperantes en el Partido Comunista, la organización más influyente de la época. La evaluación de la revista hecha por el historiador es las siguiente:

La evolución de Historia y Sociedad refleja los cambios de la vieja izquierda marxista. El primer periodo de la historia de la revista se inicia en 1965, cuando la nueva dirección del РСм acordó apoyar una revista cultural socialista, sobre el modelo de la influyente revista soviética Ciencia y Sociedad [...] Inicialmente Historia y Sociedad se propuso difundir textos soviéticos, de Europa del Este y latinoamericanos. Había escasas colaboraciones mexicanas reflejo del reducido tamaño de la academia marxista mexicana a principios de los sesenta. Ade- 
más de los tres escritores marxistas más conocidos en México, Elí de Gortari, José Revueltas y Enrique González Rojo, los dos últimos eran particularmente inaceptables para una revista que tenía estrechos vínculos con el Partido Comunista (Carr, 1996, pp. 244-245).

Las anotaciones hechas por el historiador son relevantes, pues trazan las principales líneas conflictivas por las cuales avanzó la publicación. Ahora bien, otro dato significativo tiene que ver con la independencia que el comunismo mexicano fue conquistando en esta época. Si comparamos a grandes rasgos las trayectorias de partidos comunistas de la región como el chileno o el argentino es notable el grado de independencia que su hermano mexicano logró hacia el final de los años sesenta. Pongamos por caso, la reacción ante los sucesos de 1968, cuando las tropas del Pacto de Varsovia invadieron la ciudad de Praga. Así, mientras el PCM se posicionó críticamente ante aquel acontecimiento -como lo haría el partido sucesor años después con la invasión soviética a Afganistán y en respuesta a la cercanía de un sector de la dirigencia comunista local con la vertiente del eurocomunismo- en los casos de Argentina y Chile se impuso una lógica distinta, al encontrarse aquellos países en medio de férreas dictaduras, aunque en posiciones totalmente contrarias el uno del otro.

Hasta el momento contamos con los valiosos datos aportados por Carlos Illades (2012) en su estudio sobre el papel de la izquierda en el debate público. En dicho trabajo se especifica información relevante de los primeros directores de la revista, las condiciones de producción y la intersección de su aparición dentro de las disputas internas al seno del PCM. Sin embargo, lo que nos interesa resaltar es que sin dejar de ser una revista anclada a una militancia específica, la revista fue algo más que una publicación partidaria, dice Illades (2012):

...dirigida por Enrique Semo Calev (Sofía, 1930). Roger Bartra Muriä (Ciudad de México, 1942), miembro del РСм al igual que Semo, lo acompañó como Jefe de redacción durante el primer periodo, a la vez que Boris Rosen y Froilán Manjarrez formaron parte del comité de redacción, al que se sumaron posteriormente Daniel Cazés, Alberto Híjar, Cecilia Rabell, Madalena Sancho, Raquel Tibol, Raúl González Soriano. La nueva publicación reunió a profesionales de distintos campos de las ciencias sociales y de las artes e intentó renovar la disciplina histórica recurriendo a un paradigma alternativo al empirismo e historicismo entonces dominantes (p. 51). 
En definitiva, el nacimiento de Historia y Sociedad marcó un hito, pues aunque respondió a la militancia comunista abrió paso a comunistas sin partido como Alberto Híjar o a militantes como Raquel Tíbol que no pertenecieron de manera directa, aunque gran parte de su actividad teórica y política giraron en torno a la vida política y cultural del PCM (Concheiro y Pacheco, 2016, p. 29), así como también a marxistas de la talla de Adolfo Sánchez Vázquez. De manera que Historia y Sociedad es una composición plural, animada por comunistas locales, atravesada al calor de cierta dependencia ideológica y política con el hermano soviético, pero también en un proceso de acercamiento a otras vías que reclamaban cierta autonomía. El coctel se predispone ya como tenso, tanto en las coordenadas de su producción como en el conjunto de temas que serán el elenco principal.

\section{CERTEZAS INAMOVIBLES EN HISTORIA Y SOCIEDAD}

En su intervención a propósito de la aparición del libro de Carlos Illades el antropólogo Roger Bartra (2013) señaló que durante los años de su publicación la embajada soviética en México desempeñó un papel central en la conceptualización y elaboración de la revista. Bartra señaló el hastío de uno de los componentes que efectivamente es perceptible en Historia y Sociedad, en concreto la presencia de referencias hacia los dirigentes del Estado soviético, así como a los partidos comunistas. Efectivamente ello ocurre en algunos textos, sin embargo, es interesante destacar que las referencias o citas de ciertos dirigentes venían acompañadas de una crítica al pasado inmediato: el estalinismo.

Ya en el primer número, el conocido latinoamericanista Alperovich ${ }^{1}$ (1965) señalaba que la ausencia de estudios sobre América Latina en la URSS se debía a un conjunto de consideraciones históricas y deformaciones profesionales que habían tenido mucha fuerza durante la época previa, es decir, la anterior al deshielo iniciado en 1956. Para el autor aquella época se encontraba en proceso de superación y se apuntalaba hacia un mayor interés por los temas que se anclaban en la especificidad de la región. Aquel texto, como ha ex-

${ }^{1}$ Destacado latinoamericanista soviético, reconocido en México por sus estudios sobre el proceso de independencia y la revolución agraria de 1910. Después de la disolución del Estado soviético en 1991 continuó con su actividad académica. 
plicado la latinoamericanista Soledad Jiménez (2010) respondía a las críticas que habían hecho historiadores occidentales a la producción soviética. Los vericuetos de la producción soviética sobre América Latina en aquella época serían bien retratados por Alperovich (1995) 40 años después. La tensión estaba provocada por el eminente privilegio ideológico sobre el científico que prevalecía en algunas de las elaboraciones y que también se hizo presente en la revista en cuestión. Además de ello, sobre la investigación soviética recaían prejuicios heredaros por Karl Marx (en el caso de Simón Bolívar) o bien de las producciones de los comunistas de la década de los treinta (por ejemplo, sobre José Carlos Mariátegui), deshacerse de estos prejuicios no era tarea fácil $\mathrm{y}$ tarde o temprano hubo que dar cabida a nuevas investigaciones.

Efectivamente, a lo largo de la primera época es perceptible lo que bien podríamos denominar como una falsa sobrepolitización del espacio de la historiografía. Quizá articulando esta idea en un lenguaje clásico, podríamos decir que los artículos contenían una fuerte carga de propaganda político-ideológica revestida de cientificidad. Esto es mucho más claro en los autores soviéticos quienes además de descalificar algunas posiciones políticas, entretejen una narrativa teleológica en donde los pueblos o las clases no tardarían en entrar en la órbita del comunismo y el socialismo bajo la égida de los partidos comunistas.

Trenzar una narrativa teleológica si bien no es algo ajeno a la tradición marxista no es tampoco algo inherente. Aunque esta práctica se vuelve mucho más enfática con la fetichización del partido comunista (de cualquiera de ellos) concebido como depositario de cierta "conciencia" y por lo tanto "vanguardia política" de una clase supuestamente universal. Una formulación, por cierto, ajena a Marx en donde el partido tiene también un sentido efímero (Concheiro, 2012) pero que en la disposición discursiva del poder soviético aparecía como algo imprescindible e inamovible. Esta referencia al partido es recurrente en pocos textos, ya sea por la intervención que hacía el comité editorial como indica Bartra, o porque el sentido de los artículos era de carácter pretendidamente científico y, por tanto, aquellas reverencias salían sobrando.

Un ejemplo de esta tensión entre explicación científica y certeza política se expresa bien en el texto de V. Volski (1965) ${ }^{2}$ sobre el imperialismo y la economía venezolana. En dicho escrito se presenta un estudio sobre el papel del petróleo en la economía venezolana. Según el autor dicha mercancía era

2 Director del Instituto de América Latina de la Academia de Ciencias de la URSS. 
la "fuente de todas las desgracias" de ese país. Ciertamente, la presentación de estadísticas, datos y otras variables ayudan a demostrar el anclaje petróleo y rentista de la economía venezolana, vinculado al papel de las empresas estadunidenses y la situación nada favorable de los sectores asalariados. Sin embargo, el análisis fino que se teje sobre aquella situación también se ve atravesada por reiteraciones comunes de este tipo de textos: "El rápido crecimiento del prestigio e influencia del Partido Comunista de Venezuela que se convirtió en la fuerza del movimiento democrático y de liberación nacional" (Volski, 1965, p. 117). Si bien ello no resta mérito a la presentación del texto ni al argumento, claramente se moviliza como una certeza propagandística innecesaria que aparentaba un intento de politización.

Hasta ahora sólo nos hemos concentrado en textos publicados por autores soviéticos en la revista. Sin embargo, en el conjunto de la primera época es perceptible un elemento que también hace parte del repertorio de certezas no sólo de los investigadores soviéticos, sino del marxismo entendido como un conjunto diferenciado de discursos: el privilegio de la economía sobre otros ámbitos de la vida social. La "economía” como dinámica totalizadora puede ser rastreada por varias herencias, tanto de marxismos vueltos discursos de poder (el producido en la URSS) como de versiones más críticas. En uno u otro caso, Historia y Sociedad fue un medio de producción y difusión de conocimiento que englobó un conjunto de elaboraciones que buscaron anudar economía, política e historia, con el privilegio conflictivo de la primera. Aquello era una elección teórica de la construcción de la investigación y es perceptible en el conjunto de la revista, donde otras esferas de la vida (como la crítica cultural) apenas se asoman levemente.

\section{INNOVACIÓN, APERTURA Y TENSIONES PRODUCTIVAS}

Como se mencionó anteriormente, el presente trabajo busca hilvanar una interpretación que permita valorar con justicia la intervención de Historia y Sociedad. Esto es así porque valoramos la aportación de la revista que, ubicada en la constelación de la guerra fría se movió en la tensión entre ser un órgano de pensamiento marxista, con pretensión de cientificidad social y no una revista de opinión, a la vez que se reconoció deudora de una lógica política que la obligaba a referirse a ciertas situaciones. Es justamente por esa razón 
que es posible ubicar en la publicación una trama más compleja que la de ser sólo un vocero de los científicos sociales o historiadores de origen soviético.

En consecuencia, si las certezas de la tradición política existieron, también se manifestaron las innovaciones o novedades discursivas, tanto a nivel de la temática como del abordaje. Una de las más interesantes se refiere a la relevancia del estudio y reconocimiento central del siglo XIX. ¿Cómo era posible imaginar una transformación social de la política y el Estado si se desconocía su origen? ¿Cómo determinar las fisonomías variadas, heterogéneas y abigarradas, de las naciones si no es a partir de su origen histórico? Aquí, historia no es una construcción intelectual sino la movilización de los diversos sentidos que adquiere lo político: las clases, los espacios, las tradiciones burocráticas, las contradicciones. La historia es así la forma de anudar las tramas, dimensiones y problemáticas. De modo que expondremos de manera temática dos de ellas: en primer lugar, las que tienen que ver con la construcción histórica de naciones, así como los problemas de sus democratizaciones $y$, en segundo lugar, las que se preocupan por la inclusión de distintos personajes "históricos".

El editorial (1965) del primer número comienza con la pregunta: “¿Una revista más dedica a los problemas sociales de América Latina?” (p. 1). En aquella pregunta queda aprehendido el programa editorial de la revista que contiene sus grandes líneas de intervención, afirmando que en un tiempo convulso y de revoluciones era necesario el estudio de aquellas fuerzas que irrumpen planteando las luchas por la liberación nacional. En este contexto es que la revista, según dicta el editorial (1965), busca: "contribuir en forma modesta a la presentación de materiales que abonen a los temas históricos y sociales en función del mundo cambiante" (p. 2). El editorial también señala que se dará énfasis a las investigaciones realizadas en el campo socialista, es decir, que sería un espacio donde los cientistas sociales e historiadores de la Unión Soviética y la Europa del Este tendrían un lugar central; esto sin duda se verificó con intensidad en la primera etapa de vida de la publicación. Es importante entonces marcar que las líneas de intervención que encontraremos se mueven entre la impronta sovietista o prosoviética y la necesidad de producir un conocimiento adecuado, situado e histórico sobre los procesos políticos latinoamericanos. No era la coyuntura, sino la historia lo que primaría en la primera época de la revista.

El historiador soviético Alperovich (1965), anteriormente referido, presenta un intento de sistematización del despliegue del estudio de Amé- 
rica Latina en la URSS. En su documento critica ferozmente las primeras producciones tras la revolución de 1917 que en su opinión contienen "graves errores de hecho y de teoría" (p. 59). Debido a esto, Alperovich prefiere centrarse en el periodo entre 1953 y 1963, dentro del cual logra identificar los motivos por los cuales la historia latinoamericana recibe mayor atención, estos son, por un lado, los distintos congresos del Partido Comunista de la URSS que ponen mayor énfasis en esta región del mundo y, por el otro, la emergencia de movimientos de liberación nacional que obligan a replantear la llamada "historia universal" que se enseñaba comúnmente en la URSS. En una exposición lineal el autor nos muestra el contenido de las investigaciones que refieren a los pueblos existentes previos a la conquista, posteriormente los trabajos que ponen énfasis al papel de Cristóbal Colón y el esclavismo, pasa a la época colonial y finalmente al estudio de los procesos de independencia en el siglo XIX: "Sin embargo hasta el XX Congreso del Partido Comunista de la Unión Soviética, la historiografía soviética no estuvo en grado de realizar un detallado estudio científico de los más importantes problemas de las guerras de independencia en Latinoamérica, debido a las tesis erróneas y a las apreciaciones sectarias que durante muchos años dominaron nuestra literatura histórica" (Alperovich, 1965, p. 66), en clara crítica del pasado reciente, pero también rindiendo tributo a la dirigencia posdeshielo.

El recuento pormenorizado que hace Alperovich de la producción soviética se encuentra cruzado por varias tendencias perceptibles con claridad en su escritura: en primer lugar, la inscripción de la nueva historiografía dentro del proceso de desestalinización (nombrado como "culto a la personalidad") que por fin buscaba desbloquear algunos de los prejuicios ideológicos más fuertes; en segundo lugar, la crítica a los trabajos que se habían anclado en la perspectiva política de la época stalinista que subvaloraban el papel de las elites y la burguesía o caracterizaban al populismo como fascismo; en tercer lugar, una predilección por el estudio de las revoluciones, tanto la mexicana como la cubana, y por tanto un intento de realizar la "génesis" de aquellos procesos; finalmente, la certeza de que en el estudio del movimiento obrero de los distintos países se encontraba el secreto de la liberación nacional, motivación principal de movilización en la región. La manera en que se amalgamaban los problemas sugiere un intento de nuevas hipótesis con respecto a los sujetos del cambio político, a las formas de interpretación de dichos cambios y sobre el lugar de la historia de América Latina en el conjunto de la historia global. En gran medida el texto de apertura de la revista, aun con sus 
limitaciones, marcó las posibilidades de ruptura a partir de nuevas hipótesis de interpretación. En adelante veremos la manera en que ello se realizó, tanto a nivel de procesos de constitución histórica de las sociedades como al nivel de los personajes e intelectuales que nutrieron las venas libertarias.

Será pertinente entonces comenzar por el número 4 de Historia y Sociedad como ejemplo de esta disposición de comprensión del siglo XIX, caracterizándolo como el periodo de la conformación de los Estados y con esto en gran medida de las clases sociales. Así, aquel número presenta un texto de Rodolfo Cerdas Cruz $(1965)^{3}$ al respecto de la trayectoria de este proceso en un país centroamericano por demás peculiar, como lo es Costa Rica. Cerdas Cruz construye toda una narrativa del nacimiento del Estado a partir de 1821 sobre la base de los dispositivos de clase y de división política a partir de las regiones que se confrontan permanentemente. Desde esta perspectiva, la resolución de la construcción de una economía nacional se debe tanto a la persistencia de dichos conflictos regionales, como al surgimiento de nuevos clivajes clasistas. En su narración, Braulio Carrillo aparece como el primer jefe reformista del Estado: "Carrillo tuvo siempre una preocupación constante por la incorporación productiva de la masa popular, sus disposiciones legislativas se orientaron no sólo a promover el desarrollo económico, sino a cuidar, en la medida de lo posible y sobrepasando la concepción de la época, que el peso del esfuerzo y el sacrificio no recayera como es costumbre en las espaldas del pueblo" (Cerdas, 1965, p. 43). Siguiendo su argumento la caída de Carrillo definiría finalmente la construcción del Estado nacional en Costa Rica a partir de una lucha doble: en contra de la oligarquía y en contra del pueblo.

En la misma órbita de entendimiento sobre procesos políticos de larga data el número 4 incursiona sobre dos personajes que, aunque disímiles, son fundamentales para entender la configuración del espacio centroamericano y caribeño, posicionado en la escalada geopolítica de la expansión estadunidense en el siglo xx. Por un lado, se dio apertura a una reivindicación de la figura de César Augusto Sandino de la pluma de N. Larin y, por el otro, se permitió un acercamiento crítico a la figura de Rafael Leónidas Trujillo. Así, Nicaragua y República Dominicana son incorporadas en Historia y Sociedad. Como es de esperar se resalta el papel positivo de Sandino y se explica su apa-

${ }^{3}$ Costarricense. Estudió en Francia y en la Unión Soviética, en donde se doctoró en Filosofía. De regreso a su país tuvo una intensa vida académica, política y periodística. Militó en el Partido Comunista de Costa Rica, hasta que se inclinó por las opciones maoístas. 
rición política a partir de la intervención estadunidense, pero junto a ese elemento también se advierte la debilidad de la clase obrera y la fortaleza de los latifundistas que les permitió imponerse como sostén del dictador Anastasio Somoza. Mientras que en el caso del dominicano Trujillo la explicación recae nuevamente en la intervención de Estados Unidos y los intereses geopolíticos perseguidos desde finales del siglo xIx, en tanto que la figura es retratada como la de un arribista y un personaje más bien mediocre. Personajes tan disímiles y en trincheras opuestas, son inexplicables sin considerar el papel de Estados Unidos y el conjunto de factores socioeconómicos que les permitieron relucir en naciones donde el proletariado era débil o inexistente; esto último es lo que explica los tratamientos específicos de ambos casos.

Es así que Larin $(1965)^{4}$ ve en Sandino a "el representante más genuino del movimiento de liberación nacional" (p. 73). Lo que más se destaca de "liberador" en la práctica Sandino, se contrasta con la perspectiva del gobierno trujillista, que modificó incluso el nombre de la capital: "La manía de grandeza de Trujillo era tan enorme que es muy difícil encontrar en la historia otro caso comparado al suyo" (Enánova, 1965, p. 83). Además de lo ya dicho resulta sugerente notar que los argumentos críticos al régimen de la dominicana se construyen sobre la información elaborada en folletos del Partido Socialista Popular y de la revista Life (para referirse al caso de secuestro y asesinato del académico de origen español Jesús Galíndez en Nueva York), conocida publicación estadunidense, además del acreditado texto La era Trujillo de Jesús Galíndez publicado en Santiago de Chile. En cambio, no aparece ninguna mención a las "hermanas Miraval" quienes junto a sus compañeros construyeron una oposición significativa al régimen trujillista, lo que años después las convirtió en iconos de aquella historia. En cambio, para el caso de Sandino las fuentes utilizadas fueron conocidos textos de Gregorio Selser, testimonios del español Ramón de Belausteguigoitia y de libros producidos en Estados Unidos sobre el área Caribe, al igual que informes del Departamento de Estado sobre Nicaragua.

Casi al finalizar la primera época de la revista existe otro número en donde Centroamérica vuelve a aparecer como tema de reflexión, me refiero al número 15, correspondiente a enero de 1969, en donde el costarricense Eduar-

${ }^{4}$ No existen datos del autor. 
do Mora $(1969)^{5}$ expone el lugar que tiene dentro de la economía capitalista mundial el conjunto de países centroamericanos. La hipótesis del autor es que Centroamérica ejemplifica de manera clara el proceso de integración económica, entendiendo esta como la supeditación a la economía estadunidense por la vía de la influencia de los monopolios. Rastreando y diferenciando los distintos momentos de unidad (en la colonia bajo la administración española, pero sobre todo en el periodo posindependentista) el autor busca problematizar el entramado económico construido tras el fin de la segunda guerra mundial, con respecto al papel de las clases dominantes, la debilidad de la industria y en general todos los beneficios a favor de las inversiones por parte de los sectores monopólicos. Todo ello le lleva a aclarar casi al final del texto: "Cuando nuestro Partido elevó su voz contra los proyectos imperialistas de Integración Centroamericana, no fue comprendido debidamente por algunos sectores revolucionarios del istmo. Estos creían notar en nuestra posición falta de entusiasmo para la unidad fraternal de los pueblos centroamericanos e incomprensión de los nuevos procesos observados en el mundo" (Mora, 1969, p. 64). La narración de Mora tiene el recurso de la historia para justificar una posición política contemporánea que desestima los intentos vigentes en aquel momento de la integración económica al considerarla subordinada. Así, el denso artículo de Mora apuntala la economía como el corazón de los dilemas políticos centroamericanos, por variados que estos fueran.

Continuando por la senda de la integración centroamericana es de destacarse la incorporación en el número 7 del texto de Manuel Galich (1966) sobre la cruzada de Juan Rufino Barrios en 1885. El argumento del historiador guatemalteco es que Barrios tuvo el mérito de elegir la ideología revolucionaria de su tiempo (el liberalismo) para deshacerse de las últimas rémoras del colonialismo. El intento del militar y político guatemalteco de unificar a toda la región centroamericana en una sola nación se vio frustrado cuando Estados Unidos se lanzó sobre Nicaragua con el fin de trazar la ruta comercial más importante de la época. Para Galich, Barrios era un antiimperialista con pleno derecho que pagó con su muerte el atrevimiento de querer conservar la soberanía centroamericana. El texto, de calado historiográfico, pretende advertir al lector de la época sobre la importancia de la región y alerta par-

${ }^{5}$ Reconocido dirigente comunista en Costa Rica. Fue diputado en dos ocasiones y un investigador de temas económicos desde los años sesenta. 
ticularmente de la pesada losa que carga Nicaragua, al ser un país que está constantemente en la mira de Estados Unidos.

Para el verano de 1966 el número 6 de la revista aporta dos perspectivas que valen la pena para reforzar nuestro argumento de la tensión productiva presentada en la revista, no en términos de construcción del devenir histórico de las sociedades, sino de personajes incómodos o anteriormente excluidos de la tradición comunista. Se trata de dos aportes a propósito de pensadores de alta talla dentro de la tradición latinoamericanista: José Carlos Mariátegui y Frantz Fanon. La referencia a estos personajes resulta sorpresiva, ya que entre la izquierda marxista es bien conocida la calificación de "populista" que algunos intelectuales soviéticos hicieron a principios del siglo xx del peruano Mariátegui, en tanto que el martiniqués Fanon aparece distante a la tradición comunista y, como se observará en el texto, esa distancia se confirma; sin embargo, es importante anotar la forma en que aparece el distanciamiento.

El soviético Korionov $(1966)^{6}$ firma el artículo dedicado al amauta titulado "Mariátegui: destacado marxista-leninista latinoamericano". Según este autor el peruano habría llegado al marxismo después de vencer importantes influencias por parte del anarquismo y el sindicalismo, siendo así el fundador del Partido Socialista que, según reconoce el autor, sólo tras la muerte de Mariátegui en 1929 se convierte en Partido Comunista. Si bien elude la resistencia de Mariátegui al proceso de bolchevización emprendido por la Internacional Comunista de la época, carga sus baterías contra las acusaciones hechas por intelectuales soviéticos de los años treinta indicando que "los dogmáticos a principios de los años 30 'apartaban' a Mariátegui del marxismo y lo tildaban de 'populista' solamente porque el gran marxista peruano consideraba la comunidad indígena en el Perú como un factor que puede aligerar el paso del país al socialismo" (Korionov, 1966, p. 4). El artículo es una clara muestra de la distancia -no la única que ya se estaba produciendo- que se tenía con las calificaciones negativas que se habían hecho a personajes relevantes de la vida política latinoamericana. Desde el punto de vista del autor, el énfasis en la persistencia y vitalidad de la comunidad indígena era un claro aporte de Mariátegui, quien se desmarcó de la "vía europea" o "rusa" para pensar la construcción socialista a partir de condiciones concretas. Si bien se trata de

${ }^{6}$ Formó parte del grupo de investigadores soviéticos que rectificó los juicios negativos sobre Mariátegui. Se desempeñó también como colaborador del diario oficial Pravda. 
un texto lleno de lugares comunes sobre el "marxismo-leninismo", Korionov tiene el mérito de identificar en la figura de Mariátegui un autor original desde el cual mirar diferentes posibilidades de comprensión del marxismo. El caso de las comunidades indígenas es ya un adelanto frente a las visiones exclusivamente obreristas o economicistas. Estas tesis del autor soviético serán criticadas por un reconocido estudioso de la obra de Mariátegui como lo es Arturo Melis (1979, p. 10), quien no desestimó la impronta anarcosindicalista del amauta.

Además de ello es importante señalar que el número 10 publicado en el verano de 1967 de Historia y Sociedad estuvo dedicado al 50 aniversario de la revolución rusa, del que llama la atención la portada con la imagen de Lenin, así como la inclusión de textos de autores soviéticos sobre el tema. Más aún, es de nuestro particular interés indicar que el texto que abre la revista es justamente uno de José Carlos Mariátegui. El gesto de los editores es claro, si bien no se excluye la presencia soviética de la reflexión de las cinco décadas de revolución rusa, es Mariátegui quien encabeza el número, lo que subraya la preocupación por ensayar una vía de comprensión propia y crítica desde el mirador latinoamericano.

Como se puede observar, durante estos años el pensador peruano comienza a ocupar un lugar distinto del que le había asignado el canon soviético. Así, Mariátegui progresivamente fue reivindicado como un intelectual latinoamericano de talla excepcional. Como resultado, el rescate de su figura sirvió como estrategia en contra de la ortodoxia de antaño, a la vez que contribuyó a alimentar la veta latinoamericanista de la revista.

Esta situación hace que la presencia de Mariátegui contraste con la que tuvo el hoy afamado teórico martiniqués, Franz Fanon, apenas muerto en 1961 y cuya obra fue soterrada dentro de las construcciones teóricas producidas desde América Latina. Su pensamiento fue criticado por marxistas de la época -como Francisco Delich desde Argentina-, apropiado por la izquierda "nacional-popular" argentina de la mano de Carlos Fernández Pardo, y también fue bien recibido por los marxistas caribeñistas entre quienes destacaron Roberto Fernández Retamar desde Cuba y el entonces militante trotskista Adolfo Gilly, quien desde México introdujo sus ensayos en idioma inglés. Sin duda es urgente realizar una cartografía de la recepción y apropiación de Fanon en América Latina que, motivada por la cantidad de relaciones triangulares que supuso su presencia, logre estudiar la conexión de diversas tradiciones a partir del imperativo fanoniano de pensar los problemas de la 
violencia y la cultura nacional. Es así que, en Historia y Sociedad, de manera intempestiva y crítica, aparece la figura de Fanon. Según el autor Irme Marton (1966), ${ }^{7}$ Fanon permite profundizar en tres momentos del establecimiento del orden colonial, a saber: la violencia colonizadora, la interiorización de ella y la violencia descolonizadora. De todo ello, el autor reflexiona categóricamente: "Para Fanon, el mundo colonial no es más que una confrontación de violencias que se objetivizan y de violencias que se subjetivizan" (p. 58). No es la única ni la menor de las críticas que el texto lanza contra Fanon.

Asediemos ahora otras de sus críticas. En el argumento de Marton, Fanon es un teórico que busca formular un socialismo específico y exclusivo para el tercer mundo. En ese proceso de producción teórica Marton aduce que Fanon comete el error de desplazar a la clase obrera (y con ella a la ciudad, a la burguesía nacional y a la tecnología) de un horizonte de construcción de otra sociedad distinta a la capitalista. De ahí que para Fanon la nación a construirse sólo tendría vitalidad en el campo, por consiguiente, los campesinos debían aliarse con los intelectuales y no con la clase obrera. Para Marton (1966) todas estas apreciaciones que achaca a Fanon son producto de una visión subjetivista de la historia y de un reemplazo: "de la dialéctica de las fuerzas productivas y de las relaciones de producción, de la economía y de la política, por la dialéctica de la violencia" (p. 75). Junto a esta evaluación global se suceden otras: la falta de un carácter del Estado, su silencio sobre el régimen de propiedad y en general una mayor definición en torno a los problemas de la técnica. Además de ellos el principal argumento es que Fanon representa una cierta intelectualidad de la pequeña burguesía, que puede ser efectivamente no capitalista, pero sólo a condición de la existencia histórica del campo socialista. Eludir la existencia de la técnica moderna, del socialismo y del proletariado, es lo que Marton (1966) categoriza como una visión subjetivista, la cual: "hace que Fanon busque una solución original desde todo punto de vista, que vuelva la espalda a la historia, que transgreda las leyes generales del desarrollo social y abra puerta a mistificaciones que perturban la conciencia de las masas populares, desarman su acción, permiten la infiltración de ideologías reformistas a las cuales el mismo Fanon desea oponerse” (p. 82).

${ }^{7}$ Estudioso de la obra de Frantz Fanon, publicó varios artículos exponiendo sus tesis. Entre los estudiosos de la obra del martiniquez es importante un discurso dado ante el memorial de Fanon en los años ochenta. 
La tensión es clara: el estudio de la obra de Fanon se presenta para Marton como imprescindible para entender la situación colonial y los horrores que ella genera, pero desde el punto de vista del autor ese mismo estudio demostraría la insuficiencia de los procesos "nacionalistas" que conducen por vías "originales" y no socialistas, a los movimientos anticoloniales. Más aún, esta interpretación reafirma que el "marxismo-leninismo" es la única teoría que se asienta sobre leyes históricas férreas y universales, de modo que a Fanon, ajeno a dicha órbita, se le acusa de "subjetivista". En suma, la evaluación de Marton traza los aportes de Fanon, pero su lectura desde el horizonte teleológico de la ciencia de la historia "marxista-leninista" lo lleva a desechar lo más original e importante de la obra fanoniana.

El legado de recepción y discusión de personajes antes excluidos (Mariátegui) o incorporados críticamente (Fanon) en Historia y Sociedad, contrasta con la apropiación de otro pensador que rompe los esquemas de construcción de la ideología socialista y comunista, nos referimos a fray Bartolomé de las Casas. Es así que, en el número 5 no sólo se presenta la silueta del conocido fraile en la portada, sino que prácticamente el número en su conjunto se dedica a discutir su figura. Decidimos exponer el material referente al fraile después de referirnos a las figuras del peruano y el martiniqués porque en él resulta mucho más evidente la ruptura con las formas tradicionales de entender la genealogía de los procesos de liberación. Porque Las Casas rompe los esquemas de la concepción que tematiza la impronta comunista anclada exclusivamente en el industrialismo, la ideología del progreso y en general de una serie de inspecciones decimonónicas, de las cuales Marx hace parte en más de una ocasión. De ahí que su estudio no sólo permite abonar hacia una comprensión distinta del legado de una figura tan relevante para la historia latinoamericana, sino que además permite anclar la herencia emancipatoria más allá del siglo XIX.

Es así que el número 5, aparecido en el año de 1966, jugó en la convergencia tanto de una ciencia histórica politizada que buscó la "génesis" de las luchas contemporáneas en el pasado, como en la construcción de referentes más plurales, que no estuvieran limitados a los propios de la Europa decimonónica. Concretamente, desde el editorial del número 5 (1966) queda clara la posición que se buscó problematizar a propósito de Las Casas: "Los marxistas latinoamericanos podemos estar orgullosos de ser los herederos de las tesis que expresó Las Casas en ese documento y de la digna posición histórica adelantada en varios siglos a su época" (p. 5). 
El tono estaba claramente delineado; en efecto, Las Casas era una figura digna de reivindicarse y el conjunto de trabajos incluidos en este número lo hicieron en distintas medidas e intensidades. La revista abre con el artículo del historiador soviético S. Serov (1966) sobre la presencia de Las Casas en la obra del también historiador estadunidense Lewis Hanke. El soviético somete a crítica las "tesis idealistas" de Hanke, para quien la historia sería, según Serov, el conjunto de un par de voluntades individuales. Sin embargo, también se destacó que el estadunidense colocó la figura de Las Casas como un jurista que buscó la justicia, ubicándolo así como un humanista propio del renacimiento español.

Por su parte, Juan Comas (1966) realizó una defensa historiográfica de Las Casas en contra de quienes lo demeritaron por su labor de descripción sobre la situación indígena o por algunas de sus posiciones con respecto a las poblaciones negras. Así pues, Comas realizó una a los detractores que negaron las cifras que Las Casas presentó y a aquellos ubicados en la línea que sostiene la "leyenda negra", es decir, que presentaron a un Bartolomé de las Casas esclavista. En definitiva, para Comas aun a pesar de las críticas, Las Casas es y seguirá siendo el "Protector universal de los indios".

Al mismo tiempo, el número que ahora comentamos incluye cuestiones que probablemente ejemplifican temáticas más sustanciales, las cuales dan muestra de la tensión productiva que advertimos al inicio. Por ejemplo, el historiador soviético Grigulevich ${ }^{8}$ (1966) presentó el texto titulado "Fray Bartolomé de las Casas enemigo de los conquistadores", posiblemente uno de los documentos que en mejor forma presenta los intentos de apropiación de una figura que necesariamente remite a registros distintos de los tradicionales en la órbita comunista. Una cita podrá mostrar a lo que nos referimos:

El descubrimiento y la colonización de América ejercieron una influencia positiva sobre el curso del desarrollo histórico mundial; aceleraron el avance del

${ }^{8}$ Se trata de uno de los más controvertidos y famosos personajes de la guerra fría. Además de mantener una actividad intelectual y académica muy intensa (que incluyó la publicación de múltiples trabajos) Grigulevich fue un espía. Actúo en diversos frentes en Europa, siendo la guerra civil española uno de los más controvertidos. En América Latina logró infiltrarse en el servicio diplomático de Costa Rica, en donde fue encargado de Negocios en la embajada de Yugoslavia, en donde se supone que trabajaba en una conspiración contra el mariscal Tito. La reconstrucción de las "cinco vidas" de Grigulevich se encuentran en el trabajo de Marjorie Ross (2004). Además de esto publicó libros sobre temas latinoamericanos muy diversos, así como biografías de Pancho Villa, Salvador Allende, el Che Guevara, entre otros. 
modo de producción capitalista y fortalecieron las posiciones de la burguesía en una serie de países europeos con los que se aceleró el hundimiento del feudalismo en España. Sin embargo, para las poblaciones aborígenes americanas, la conquista significó una inmensa desgracia, cuyas consecuencias se perciben aún hoy (Grigulevich, 1966, p. 43).

Este fragmento permite observar la tensión entre la reivindicación de la figura de Las Casas y la manera de captar el devenir histórico de la aparición de América en la historia. No obstante, es perceptible que el texto tiene primordialmente una intensión informativa antes que una de debate, pues presenta extensamente los datos biográficos del personaje en cuestión y solamente se detiene a referir las opiniones de Las Casas al respecto de la conquista, las cuales hacen parte de la última etapa de la actividad teórica del dominico. El historiador soviético remata: "el que se compare a un sacerdote con los comunistas puede resultar a primera vista, paradójico, aunque existen motivos que hacen racionalmente válida la comparación" (Grigulevich, 1966, p. 46). En resumen, el argumento del soviético se encaminó por el sendero que valida esta sentencia, al reconocer a esa figura icónica como antecedente de las luchas contemporáneas de América Latina.

En una senda similar, aunque más actualizada, se desarrolló el texto del también soviético Zubritski" (1966) quien escribió "De la 'protección de los indios' del padre Las Casas al indigenismo contemporáneo”. En dicho artículo, el autor buscó desentrañar las diversas facetas del indigenismo, al que considera un fenómeno contradictorio y complejo, cuyo origen se remonta a finales del siglo xix y que tuvo en Manuel González Prado y en Florinda Matto de Turner dos cabezas excepcionales. De ahí, pasa a realizar una crítica a los esfuerzos que denomina "reaccionarios", como aquellos que se desprenden de los Congresos Indigenistas Interamericanos -el primero de ellos fue celebrado en Pátzcuaro, Michoacán, en 1940-y en general de la Organización de Estados Americanos, quienes se empeñaron en ser los "protectores" de los pueblos indígenas:

${ }^{9}$ Antropólogo soviético, especialista en temas de culturas indígenas. Durante los años setenta tuvo mucha influencia en el área de la lingüística. Existen varios artículos publicados en Ecuador y en la revista América Latina que se publicaba en Moscú. 
No obstante, el Instituto Indigenista Interamericano y otras entidades indigenistas no son absolutamente un dócil instrumento del Departamento de Estado. Más bien se les puede caracterizar como un campo de lucha tenaz, aunque con frecuencia velada y sorda, entre los indigenistas progresistas y los que enarbolan la bandera del indigenismo como biombo tras el cual se esfuerzan en agrandar la prepotencia imperialista en Latinoamérica (Zubritski, 1966, p. 57).

El autor continúa sus críticas no sólo a las misiones dependientes de Estados Unidos, sino también hacia al concepto mismo de aculturación y a los "indigenistas" dependientes de oficinas estatales como los promotores de dicha política, a la que él ve como la responsable de la destrucción de las culturas. Todo ello le sirve como material para contrastar la actitud del padre Las Casas, que aprendió la cultura y no buscó erradicarla. Así, por ejemplo, contrasta: "Alejandro Lipschutz no se proclama continuador de Bartolomé de las Casas, pero los hombres de su talla merecen llamarse justamente continuadores de todo lo positivo y valioso que deja en pos de sí el fraile español" (Zubritski, 1966, p. 63). Su conclusión es que el campo de batalla del indigenismo se bate entre los "asimilacionistas" que sirven sin quererlo a veces a la política estadunidense y los revolucionarios que apuestan al desarrollo de los pueblos indígenas. Dentro del segundo grupo, es importante la mención del antropólogo chileno Alejandro Lipschutz, un autor que destaca la viabilidad de la teoría de Marx y la de Lenin para analizar a las poblaciones indígenas de la región.

En el mismo tenor hay que señalar que en el número 10 de Historia y Sociedad el entonces joven antropólogo Daniel Cazés presentó un conjunto de reseñas críticas sobre trabajos recientes y en español dedicados a Las Casas, reunidos todos en una obra titulada Estudios Lascasianos. La reseña de Cazés es en tono de especialista y no hila necesariamente-como los trabajos presentados en el número 5- el papel de Las Casas como político de manera inmediata. Sin embargo, es conveniente señalar el ejercicio de Cazés para mostrar una continuidad en la proyección de la figura del dominico, más allá del número monográfico que presentamos en extenso.

Como pudo observarse en los conjuntos señalados, en Historia y Sociedad existieron intentos de renovación interpretativa que cruzaron permanentemente con certezas e hipótesis clásicas. Como resultado, las incorporaciones de personajes relevantes fuera del mundo comunista tradicional fueron 
acompañadas de silenciamientos importantes, es así que junto a las figuras históricas y los procesos clave de construcción nacional, pervivió la tendencia al privilegio de la economía, aunque no siempre de una manera reduccionista. Son estos algunos ejemplos de eso que hemos denominado una tensión productiva, la cual nos permite considerar la publicación en cuestión como algo más que una correa de transmisión de la ciencia social e histórica soviética.

\section{OTRAS VETAS DE ANÁLISIS}

Es posible enriquecer lo expuesto hasta aquí apuntalando algunos temas sugerentes para la crítica cultural latinoamericana. Nos referimos en específico a la cuestión artística, que se abrió camino en Historia y Sociedad. Dos de los críticos de arte más importantes de México participaron en sus páginas y expresan una veta que el comunismo mexicano siempre consideró importante. Tanto Raquel Tibol como Alberto Híjar sostuvieron una importante presencia en este rubro de la revista. Tibol, una estudiosa a profundidad de las obras de David Alfaro Siqueiros, Frida Kahlo y Diego Rivera, entre otros, era ya una reconocida crítica de arte y figura reconocida en el PCM (Concheiro y Pacheco, 2016). Híjar era por entonces un joven filósofo, muy cercano a Siqueiros y un comunista sin partido. Su obra desplaza la noción idealista de "historia del arte" (como un cúmulo de periodos que se suceden) por la de "protección artística" (Esquivel, 2016, p. 28). Esta última categoría pretende acceder a una comprensión materialista, es decir, busca comprender la dimensión artística en cuanto trabajo que se asienta sobre determinadas condiciones técnicas, lección que como se verá adelante, proviene del estudio de la obra de Siqueiros.

La temática artística se expresó de diversas formas en Historia y Sociedad. Una fue el comentario sobre el trabajo de algunas figuras relevantes. En gran medida su participación política y su innovación estética son los elementos que aparecen como elementos centrales para la valoración positiva. También se hará referencia a trabajos de orden teórico.

Así, en el número 2 de la revista se presenta un texto de Tibol en donde expone a propósito de José Guadalupe Posada, señalando de entrada un rasgo que le es útil para evaluar su obra: "Dime a qué público sirves y te diré qué arte produces" (Tibol, 1965, p. 97). Sirviéndose de algunos conceptos del filósofo Jean Paul Sartre, Tibol ubica a Posada en el despliegue histórico de su mo- 
mento, del papel de las clases en el desarrollo histórico y del lugar que tenían a principios de siglo. Señala que fue la ineptitud de las clases dominantes la que permitió a Posada generar un discurso artístico libre. El análisis de Tibol apunta entonces a ubicar las coordenadas de desarrollo capitalista, en donde el grabador desempeña un papel que no necesariamente eligió, sino que se encontró en medio de algunas condiciones que se tornaron adecuadas para desarrollar su obra. Así "La obra de Posada constituye el primer rompimiento con el colonialismo cultural; su obra es el precedente más importante de la revolución artística" (Tibol, 1965, p. 100). Ello es así, concluye la crítica, porque Posada retrató al "populacho" en el que vivió, mismo que fue el actor central en la revolución de principios de siglo.

En el número 3 aparece una reseña de Híjar sobre el libro de Adolfo Sánchez Vázquez Las ideas estéticas de Marx. Híjar, ayudante de la cátedra de Estética que Sánchez Vázquez dictaba en la Facultad de Filosofía, presenta "Las ideas estéticas de Marx" como un ejemplo del tratamiento renovado de las ideas del filósofo alemán. Destaca los distintos niveles en los que se mueve el análisis del filósofo trasterrado, que van de la exploración original de los textos juveniles hasta el tratamiento de cuestiones específicas como la dimensión trágica, pasando por una revisión de la obra de Franz Kafka. Híjar señala la validez que toma en la obra en cuestión la forma marxista de acercarse a la cultura, siendo este el aporte medular del libro de Sánchez Vázquez. Este tema guarda importancia pues durante algunas décadas el filósfo hispano-mexicano será un referente en América Latina de una forma heterodoxa de concebir el marxismo. Hay que señalar que no fue el único texto exclusivamente teórico que se presentó en la revista, pues en el número 15 se publica un texto de Michail Lifshit sobre "La estética en Hegel". El autor era un filósofo cercano al teórico húngaro Gyorgy Lukács, una de las fuentes predilectas de Sánchez Vázquez.

También en el número 4 se presenta un artículo escrito por Híjar sobre Siqueiros. En él se recorren las distintas etapas de Siqueiros, sus viajes y sus influencias. Destaca la manera en que el pintor recibe algunas concepciones, como la de la "raza" en el futurismo italiano (Híjar, 1965b, p. 103), de las cuales logra desprenderse. El análisis tiene su fuerte en la obra No hay más ruta que la nuestra, donde se expresa la madurez de Siqueiros. Es en ella donde se valoran con plenitud los actos de rebeldía de la plástica mexicana, que, a decir del autor, superan cualquier formato esnob o chic. Es también en esa obra en donde se hace una defensa del muralismo, no por su contenido, sino porque 
la forma misma apuntala una cierta democracia en el arte, que supera las formas elitistas, como son la pintura de caballete o el grabado numerado. El texto de Híjar busca aprender la relación que hay entre la forma marxista de comprender los cambios técnicos y su utilización en la tarea artística, lo cual sería el aporte central de Siqueiros.

En el número 7 un anexo contiene un homenaje a Siqueiros, en donde se publica un texto de su autoría titulado "Rectificaciones sobre las artes plásticas en México". En dicho texto Siqueiros reafirma una situación paradójica: que el arte puede servir a la lucha política proletaria, pero que él mismo en tanto artista, considera que la finalidad estética última se encuentra en lograr un arte puro. Sin embargo, dicha forma del arte no puede ser realizada en el capitalismo y sólo trascendiendo dicho sistema es posible aspirar a ella.

\section{CONSIDERACIONES FINALES}

Existen varias estrategias para asediar una publicación como Historia y Sociedad, la primera de ellas es la que resalta sus atisbos de originalidad con respecto a publicaciones anteriores del PCM, contando entre sus antecedentes menos afamados con la revista Teoría (publicada discontinuamente en los años cincuenta y cuyo contenido versaba más sobre la actividad política inmediata) y Nueva época (publicada durante los años sesenta). Aquellas publicaciones entendían la historia como algo directamente ligado a la política, no realizando así una separación entre una reflexión intelectual de más largo aliento y lo que distintas coyunturas políticas demandaban. Podríamos sugerir que Historia y Sociedad es la primera revista del PCM que asumió lo que el historiador Elías Palti (2005) vio como una constante de la crisis del marxismo: la pretensión de constituirse como un saber científico y su búsqueda por ser una verdad política.

Una segunda forma de asediar la publicación es la que se ha privilegiado en nuestro texto, nos referimos a un acercamiento a partir del motivo latinoamericano y/o latinoamericanista. Desde esta postura, Historia y Sociedad se distingue no sólo de las publicaciones comunistas mexicanas previas, sino también de otras publicaciones ligadas a aquella vertiente. Tuvieron que pasar algunos años para que aparecieran publicaciones con el mismo ánimo como lo fueron Dialéctica, Coyoacán y Cuadernos Políticos, todas ellas elaboradas y convocadas por los distintos exilios latinoamericanos que encontraron 
en México espacio de inserción académica o editorial de forma privilegiada. Antes que todas ellas, Historia y Sociedad sostuvo una incesante mirada sobre ese espacio, más aún, no lo limitó a la política inmediata, sino que amplió sus marcas hacia el pasado, es decir, hacia la construcción histórica de las economías nacionales o al trascurrir de la dominación de lo que se interpretó como la época de los monopolios.

Este último punto, aunque no es explícito, operó en relación con una de las certezas de la elaboración teórica de la época, nos referimos a la del entendimiento de la relación entre las naciones mediada por la categoría de imperialismo y de capital monopólico, abordada por el especialista Carlos Maya (1994), quien la explicó como un producto de los "nietos teóricos de Lenin". Habría que decir que para los historiadores soviéticos de la época además de la explicación del imperialismo y capital monopolista también se destacó la vertiente geopolítica de Estado Unidos. Pongamos por caso la extensa compilación sobre la Historia de las intervenciones norteamericanas (1982) en donde se pasó revista a las experiencias más conocidas: las invasiones a México en el siglo xIx, la guerra hispano-cubana, la intervención en Cuba, Nicaragua, Haití, Puerto Rico y Guatemala. En pocas palabras, el imperialismo no era sólo una categoría producto del desarrollo económico, sino que además se acompañó del intervencionismo como forma política.

Dentro del espesor latinoamericanista que se afianzó es perceptible el predominio de la economía sobre la política o una explicación subordinada de la segunda a partir de la primera. Ahí no podemos señalar una tendencia rupturista, pues se seguía entendiendo que la génesis del Estado, de las clases y en conjunto de las fuerzas sociales, tenía su matriz en la "estructura", es decir, lo "determinante en última instancia". Y si bien la revista mostró poco a poco apertura hacia otros temas, como la crítica cultural y artística de la mano de Raquel Tibol y Alberto Híjar, lo cierto es que en los análisis históricos la prerrogativa era clara y contundente. La tensión estaba puesta en el conjunto de la revista, pero también al seno de los análisis que se hacían en específico. Dicha tensión se mostraba en el privilegio de la economía, pero también en la búsqueda de explicaciones de más larga data. En ese sentido, al adentrarse en el siglo xIX se dejaba atrás la fácil explicación sobre la persistencia del imperialismo monopolista como fuente de todos los males, aunque esta se mantenía en los análisis de la economía del siglo xx.

Para la historia y la constitución de las naciones como objeto de estudio se abrevó de las fuerzas locales o, como diría el boliviano René Zavaleta Merca- 
do -integrante de la segunda época-, se apeló más a la forma primordial y no a la determinación dependiente. Esta tensión es clara en la revista, pero también lo fue en el tránsito del marxismo de la época, que pasó de un discurso que buscó renovarse dejando atrás las señas de ser una filosofía de la historia en clave universal a pretender un análisis "concreto de la situación concreta".

Junto a ello también es perceptible la incorporación de nuevas temáticas o de algunas que habían sido reprimidas al seno de la tradición. Nosotros destacamos en este rubro la incorporación de trabajos que abordaron figuras como las de José Carlos Mariátegui, Frantz Fanon y Bartolomé de las Casas. Dichas incorporaciones permitieron amalgamar de otra forma la construcción de las herencias ideológicas: dieron apertura a personajes que poco a poco eran reapropiados o apropiados por el comunismo. En el caso de Mariátegui, como resultado de la sintonía con trabajos de latinoamericanos, soviéticos y europeos, se logró romper el antiguo silenciamiento, aunque manteniendo una actitud crítica ante sus simpatías con Sorel y otras influencias ajenas al marxismo, como, por ejemplo, los trabajos de Kossok (1973) o Shugolvski y Semionov (1973).

Colocamos cierto énfasis en dichas incorporaciones pues suponen intentos de renovación. En el caso de Mariátegui con respecto a los anteriores momentos de crítica realizada por soviéticos. Tratándose de una revista con proyección latinoamericana es de resaltar que aún se está lejos del boom o "giro mariateguiano" que se verá en los años setenta y ochenta, donde prácticamente todos los teóricos redescubrieron, en diversos grados, el potencial de Mariátegui ya no sólo como icono, sino como motivo productivo. Sólo para mencionar los nombres de quienes pronto encontraron y explotaron la potencia de Mariátegui más allá de los debates de su época están: René Zavaleta, Oscar Terán, José Aricó, Aníbal Quijano, Alberto Flores Galindo, entre otros.

Aún más sorprendente es el caso de Bartolomé de las Casas, toda vez que obligó a los participantes a pronunciarse frente a los problemas referentes al colonialismo histórico -que nace en el siglo XVI- en su relación con el naciente capitalismo, pero también a abrevar de la problemática indígena. La complejidad de ambas constelaciones no permite que sean subsumibles al problema de la conformación de las clases y por tanto exigen ruptura dentro de la forma esquemática del marxismo. En este intento de hacer más plural la genealogía del discurso comunista, aun los soviéticos tuvieron que ampliar sus miras, como en el caso de Grigulevich (1988), quien en un texto tardío llegó a incluir a personajes como Túpac Amaru y Toussaint L'Ouverture. Pese a 
esto, consideramos que el trabajo historiográfico al respecto de los marxistas incómodos como Mariátegui o de los luchadores de la época colonial como Las Casas, en repetidas ocasiones permitió omisiones, las cuales contribuyeron a silenciar contradicciones y a pasar por alto algunos elementos que bien podrían haber sido considerados incómodos.

Caso más problemático dentro de las apropiaciones es el de Frantz Fanon. Si bien este nunca fue condenado o rechazado por la dirigencia comunista soviética, lo cierto es que fue más bien icono de vertientes más heterodoxas del marxismo. Y es que Fanon de alguna manera representa la radicalidad de una época en donde la violencia parecía ser la única alternativa. No es casual que uno de los primeros países donde se le tradujera fuera Cuba, a instancias de Ernesto el "Che" Guevara. Sin embargo, su no identificación con las instancias comunistas en Moscú o bien la distancia que el PCM sostenía con la lucha armada, pueden ser motivos suficientes para incorporar una crítica puntual al ahora acreditado pensador martiniquez.

No fueron estos los únicos temas que tuvieron desarrollo tenso en las páginas de Historia y Sociedad, como lo ha recordado recientemente Bartra, la trama de inclusión de otras temáticas - como el concepto de modo de producción asiático- causó algún revuelo en las interpretaciones consideradas como oficiales. A la par de ello habría que señalar también las renovaciones historiográficas mexicanas, de la mano del propio Enrique Semo, pero también de otros personajes que ganaron relevancia años después, siendo quizá el caso más conocido el de Enrique Florescano.

Lo que hemos ofrecido en estas páginas es una contribución inicial y parcial de una de las posibles temáticas de estudio de Historia y Sociedad. Las revistas como espacio de investigación abren puertas para entender ciertas dinámicas entre los grupos políticos e intelectuales, al ser vehículos de discusión y depositarias de tendencias y corrientes de pensamiento a los que de otra manera no podríamos acceder.

\section{LISTA DE REFERENCIAS}

Alperovich, M. (1965). El estudio de la historia de los países de América Latina en la URSS (1953-1963). Historia y Sociedad, 1, 69-86.

Alperovich, M. (1995). La revolución mexicana en la interpretación soviética del periodo de la "guerra fría". Historia Mexicana, 44(4), 677-690. 
Bartra, R. (2013). Sobre Carlos Illades, La inteligencia rebelde. La izquierda en el debate público en México, 1968-1989. Historia Mexicana, 63(1), 492-498.

Carr, B. (1996). La izquierda mexicana a través del siglo xx. México: Era.

Cerdas R. (1965). Formación del Estado en Costa Rica (1821-1842). Historia y Sociedad, 4, 37-55.

Comas, J. (1966). Los detractores del protector universal de indios y la realidad histórica. Historia y Sociedad, 5, 20-39.

Concheiro, E. (2007). Los comunistas mexicanos, entre la marginalidad y la vanguardia. En E. Concheiro, H. Crespo y M. Modonesi (comp.). El comunismo, otras miradas desde América Latina (pp. 527-558). México: СеIICH.

Concheiro, E. (2012). Reencuentro con Marx. México: CEIICH.

Concheiro, E. y Pacheco, V. (2016). La militancia crítica de Raquel Tibol. En E. Concheiro y V. Pacheco (comps.). Raquel Tibol. La crítica y la militancia (pp. 11-35). México: Cemos.

Crespo, R. (2010). Introducción. En R. Crespo (coord.). Revistas en América Latina: proyectos literarios, políticos y culturales (pp. 9-34). México: CiALc.

Editorial (1965). Historia y Sociedad, 1, 1-4.

Editorial (1966). Historia y Sociedad, 5, 3-6.

Enánova, E. (1965). La era de Trujillo. Decenios negros en la historia dominicana. Historia y Sociedad, 4, 79-93.

Esquivel, M. (2016). Alberto Híjar: lucha de clases en la imaginación. México: Cisnenegro/CenidiaP.

Fuente, J. de la (2016). Contra viento y marea: la pertinaz historia del movimiento campesino y las izquierdas. México: UACH.

Galich, M. (1966). La guerra antiimperialista de 1885 en Centro América. Historia y Sociedad, 7, 37-44.

Grigulevich, J. (1966). Fray Bartolomé de Las Casas enemigo de los conquistadores. Historia y Sociedad, 5, 40-52.

Grigulevich, I. (1988). Luchadores por la libertad de América Latina. Moscú: Progreso.

Híjar, A. (1965a). Las ideas estéticas de Marx: reseña sobre el libro de Adolfo Sánchez Vázquez. Historia y Sociedad, 3, 139-142.

Híjar, A. (1965b). Siqueiros como teórico del arte. Historia y Sociedad, 4, 102-111.

Illades, C. (2012). La inteligencia rebelde. México: Océano.

Jiménez, S. (2010). Memorias de la guerra fría: historiografía soviética latinoamericanista. Pacarina del Sur. Revista de Pensamiento Crítico Latinoamericano, 159. Recuperado de http://www.pacarinadelsur.com/home/mallas/159-memorias-de-la-guerra-fria-historiografia-sovietica-latinoamericanista? 
Kossok, M. (1973). José Carlos Mariátegui y el pensamiento marxista en el Perú. En El marxismo latinoamericano de Mariátegui (pp. 43-66). Buenos Aires: Crisis.

Korionov, V. (1966). Mariátegui: destacado marxista-leninista latinoamericano. Historia y Sociedad, 6, 1-10.

Larin, N. (1965). Sandino y la lucha liberadora del pueblo de Nicaragua contra la intervención armada de los E. U. (1927-1933). Historia y Sociedad, 4, 69-78.

Marton, I. (1966). Sobre la tesis de Frantz Fanon. Historia y Sociedad, 6, 55-83.

Maya, C. (1994). Ilusiones y agonías de los nietos (teóricos) de Lenin. México: Siglo XXI.

Melis, A. (1979). Mariátegui, primer marxista de América. México: cela-unAm.

Modonesi, M. (2003). La crisis histórica de la izquierda socialista mexicana. México: UCM.

Mora, E. (1969). La integración centroamericana, un caso de penetración imperialista. Historia y Sociedad, 15, 52-82.

Palti, E. (2005). Verdades y saberes del marxismo. Buenos Aires: FCE.

Pita, A. (2013). Reseña sobre Aimer Granados (coord.), Las revistas en la historia intelectual de América Latina: redes, intelectuales, política y sociedad. Latinoamérica. Revista de Estudios Latinoamericanos, 57, 296-299.

Ross, M. (2004). El secreto encanto de la кGв: las cinco vidas de Iósif Griguliévich. Costa Rica: Norma.

Serov, S. (1966). Bartolomé de las Casas: su vida y su obra en los estudios de Lewis Hanke. Historia y Sociedad, 5, 7-19.

Shulgovski, A. y Semionov, S. (1973). El papel de Mariátegui en la formación del partido comunista del Perú. En El marxismo latinoamericano de Mariátegui (pp. 105136). Buenos Aires: Crisis.

Tarcus, H. (2016). Para un programa de estudio sobre los marxismos latinoamericanos. Memoria: Revista de Crítica Militante, 257(1), 62-73.

Tibol, R. (1965). José Guadalupe Posada: puente entre dos siglos. Historia y Sociedad, 2, 97-106.

Volski, V. (1965). El imperialismo norteamericano y la formación de la economía venezolana. Historia y Sociedad, 2, 107-120.

Zubritski, Y. (1966). De la "protección a los indios" del padre Las Casas al indigenismo contemporáneo. Historia y Sociedad, 5, 53-65. 


\section{OTRAS FUENTES}

\section{Bibliografía}

Grigulevich, J. (ed.) (1982). Historia de las intervenciones norteamericanas, T. II. Moscú: Academia de Ciencias.

Siqueiros, D. (1966). Rectificaciones sobre las artes plásticas en México. Historia y Sociedad (suplemento), 7, v-Xv. 